\title{
Participation of CD34-enriched mouse adipose cells in hair morphogenesis
}

\author{
JING HE $^{1 *}$, HUICHUAN DUAN ${ }^{1 *}$, YAO XIONG ${ }^{1}$, WENJIE ZHANG ${ }^{1}$, \\ GUANGDONG ZHOU ${ }^{1,2}$, YILIN CAO ${ }^{1,2}$ and WEI LIU ${ }^{1,2}$ \\ ${ }^{1}$ Department of Plastic and Reconstructive Surgery, Shanghai 9th People's Hospital, \\ Shanghai Jiao Tong University School of Medicine, Shanghai Key Laboratory of Tissue Engineering; \\ ${ }^{2}$ National Tissue Engineering Center of China, Shanghai, P.R. China
}

Received October 8, 2012; Accepted January 9, 2013

DOI: $10.3892 / \mathrm{mmr} .2013 .1307$

\begin{abstract}
Adipose-derived stromal vascular fraction (SVF) cells are heterogeneous in nature, containing a number of different cell types. Recent studies indicate that CD34 may be a specific marker for adipose-derived mesenchymal stem cells (ADMSCs). Using their participation in hair morphogenesis as a model, the multi-differentiation potential of adult stem cells was investigated. In addition, adipose tissue or adipogenic lineage cells appear to be associated with the hair follicle cycle. The purpose of this study was to test the potential of CD34 $4^{+}$ cells enriched from fat tissue in hair morphogenesis. To investigate this, unsorted SVF, CD34+ ${ }^{+}$and CD34- cells sorted from the $\mathrm{SVF}$ of green fluorescence protein (GFP) transgenic mice were mixed with fetal mouse keratinocytes and dermal fibroblasts of gestational day 17.5 (E17.5) and then subcutaneously injected into nude mice. The results showed that in the reconstituted skin tissue, larger tissue blocks with more developed hair follicles were observed in the $\mathrm{CD} 34^{+}$cell group compared with the other two groups. Histological and immunofluorescent staining analyses revealed that only $\mathrm{CD} 34^{+}$cells may participate in hair morphogenesis by their integration into dermal sheath structures. However, no involvement in other skin appendages was observed. In addition, differentiation into endothelial cells and participation in blood vessel formation were also observed in both $\mathrm{CD}_{34}^{+}$and SVF cells, but not in CD34- cells. As expected, participation in adipogenesis was observed in all groups. Our results suggest that $\mathrm{CD} 34^{+}$cells may represent the ADMSCs which possess stronger multiple differentiation potential during reconstituted skin development.
\end{abstract}

Correspondence to: Dr Wei Liu, Department of Plastic and Reconstructive Surgery, Shanghai 9th People's Hospital, Shanghai Jiao Tong University School of Medicine, 639 Zhi Zao Ju Road, Shanghai 200011, P.R. China

E-mail: liuwei_2000@yahoo.com

*Contributed equally

Key words: CD34, adipose-derived stem cells, adipose-derived mesenchymal stem cells, hair morphogenesis, dermal sheath

\section{Introduction}

Adipose-derived stem cells (ASCs) are considered to be an ideal source for tissue regeneration due to their high proliferative capability and their multi-lineage differentiation potential, such as adipogenic, osteogenic and chondrogenic lineage differentiations $(1,2)$. Hair follicles are unique organs in mammals, containing multiple tissue types. Previous studies have revealed a close correlation between subcutaneous adipose tissue and hair follicle formation and function $(3,4)$.

However, it is not clear whether ASCs are capable of directly participating in hair follicle morphogenesis. In fact, several studies have revealed that bone marrow-derived stem cells (BMSCs) participate in skin appendage formation. Kataoka et al (5) have demonstrated that murine BMSCs are capable of differentiating and participating in hair follicle formation when implanted with embryonic mouse skin cells (at gestational day 17.5) in a skin reconstitution model of nude mice. Another study showed that BMSCs induced by co-culture with sweat gland cells participate in the formation of sweat glands and other skin appendages (6). Whether or not ASCs have the capability similar to BMSCs to participate in hair follicle morphogenesis has yet to be explored.

It has recently been recognized that ASCs are a heterozygous cell population containing multiple cell types, including vascular endothelial cells, pre-adipocytes, smooth muscle cells, hematological lineage cells, hematopoietic stem cells and mesenchymal stem cells (MSCs) (7). Therefore, to examine the ability of ASCs to participate in hair morphogenesis it would be ideal to use MSCs purified from ASCs. Currently, adipose-derived MSCs (ADMSCs) are considered to reside in a perivascular location and CD34 may be one of the specific markers for MSC identification and enrichment from adipose tissue $(8,9)$.

In this study, cells freshly isolated from mouse adipose tissue (stromal vascular fraction, SVF) were sorted using flow cytometry to give rise to three subgroups: i) $\mathrm{CD}^{+} 4^{+}$ (lineage $: \mathrm{CD}^{-} 5^{-} \mathrm{CD}^{-} 1^{-}: \mathrm{CD}^{+} 4^{+}$) cells; ii) CD34- (lineage CD45:CD31:CD34') cells; and iii) unsorted SVF cells. Three subpopulations were then tested for their potential to participate in hair follicle formation using a previously reported hair morphogenesis model (5). 


\section{Materials and methods}

Animals. Female C57BL/6 mice with confirmed pregnancy and 4-6-week-old male athymic nude mice were purchased from the Slac Laboratory Animal Center (Shanghai, China). In addition, C57BL/6-green fluorescent protein (GFP) transgenic mice were purchased from the Model Animal Research Center of Nanjing University (China). An Institutional Review Committee of the Shanghai Jiao Tong University School of Medicine approved all animal study protocols.

Cell isolation and flow cytometry sorting. As previously described (8), the inguinal adipose tissues were harvested from 6-8-week-old GFP-transgenic mice and then digested at $37^{\circ} \mathrm{C}$ with $0.075 \%$ collagenase (NB4, Serva, Coger, Paris, France) in phosphate-buffered saline (PBS) containing $2 \%$ bovine serum albumin (BSA) for 60-90 min. After filtration through a 40- $\mu$ m nylon filter mesh (BD Falcon, Franklin Lakes, NJ, USA) and centrifugation, the freshly isolated SVF cell portion was resuspended in PBS containing 1\% fetal bovine serum (FBS) for antibody labeling.

For cell sorting, isolated SVF cells were stained with phycoerythrin (PE)-conjugated rat anti-mouse CD34, PE-Cy5 conjugated rat anti-mouse CD45 and CD31 (1:100 in PBS containing 1\% FBS, BD Biosciences, San Jose, CA, USA) and biotin-conjugated rat anti-mouse hematological lineage cocktail (1:100 in PBS containing 1\% FBS, BD Biosciences) followed by a PE-Cy5 conjugated streptavidin secondary antibody (1:1,000 in PBS containing 1\% FBS, BD Biosciences). The cells were washed twice and resuspended in PBS containing 1\% FBS followed by fluorescence-activated cell sorting (FACS, Beckman Coulter, Miami, FL, USA) and analyzed on a FACSort with CellQuest Pro v5.2.1 software (BD Biosciences). Sorted cells were collected as the subpopulations: i) CD34+ (lineage: $\mathrm{CD} 45: \mathrm{CD} 31^{-}: \mathrm{CD} 34^{+}$) cells; ii) CD34-(lineage:CD45:CD31:CD34) cells; and iii) unsorted SVF cells. These three groups of cells were used for subsequent experiments.

Isolation of mouse fetal epidermal and dermal cells. Briefly, fetal mouse dorsal skins were harvested from C57BL/6 mouse embryos of gestational day 17.5 (E17.5), and then incubated in $0.2 \%$ dispase (Roche Diagnostics, Indianapolis, IN, USA) dissolved in Dulbecco's modified Eagle's medium (DMEM, Gibco BRL, Gaithersburg, MD, USA) containing 10\% FBS overnight at $4^{\circ} \mathrm{C}$. Subsequently, the epidermis was mechanically separated from the dermis using forceps. Then, the epidermis and the dermis were digested with $0.2 \%$ collagenase (Sigma, St. Louis, MO, USA) in PBS for $1 \mathrm{~h}$ at $37^{\circ} \mathrm{C}$. After digestion, the two dissociated cell types were washed in DMEM containing 10\% FBS and then collected and counted before grafting.

Cell grafting. The hair morphogenesis model was performed as previously described (5). Male athymic nude mice were anaesthetized by intraperitoneal injection of pentobarbital sodium ( $1.3 \mathrm{mg} / \mathrm{kg}$ body weight). Briefly, $1 \times 10^{6}$ of the CD34 (lineage: $\mathrm{CD} 45^{\circ}: \mathrm{CD}^{-}: \mathrm{CD} 34^{+}$) cells, CD34- (lineage :CD45 :CD31: CD34-) cells and unsorted SVF cells were mixed with $1 \times 10^{6}$ fetal mouse epidermal cells and $1 \times 10^{6}$ fetal dermal cells

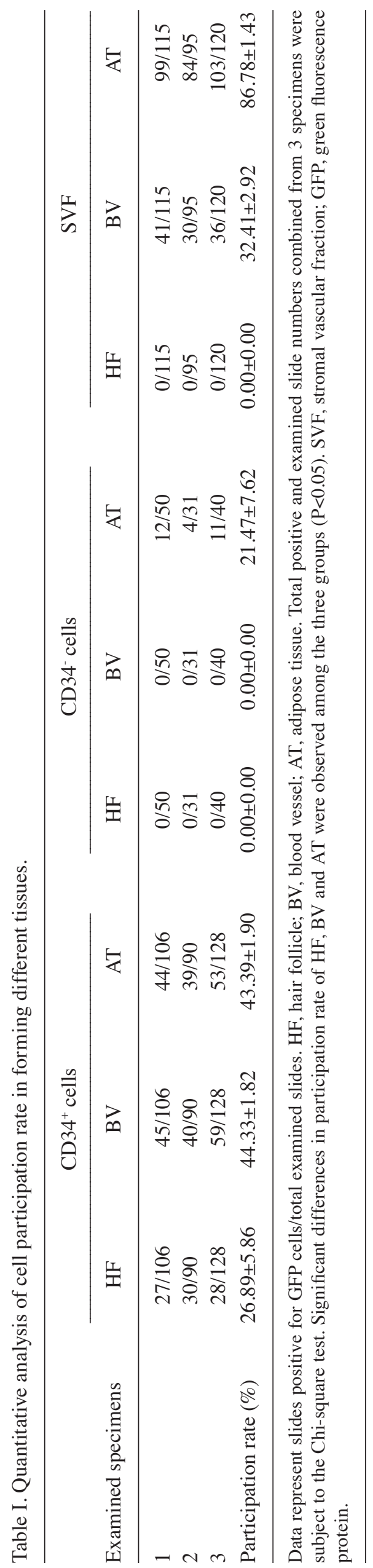


A

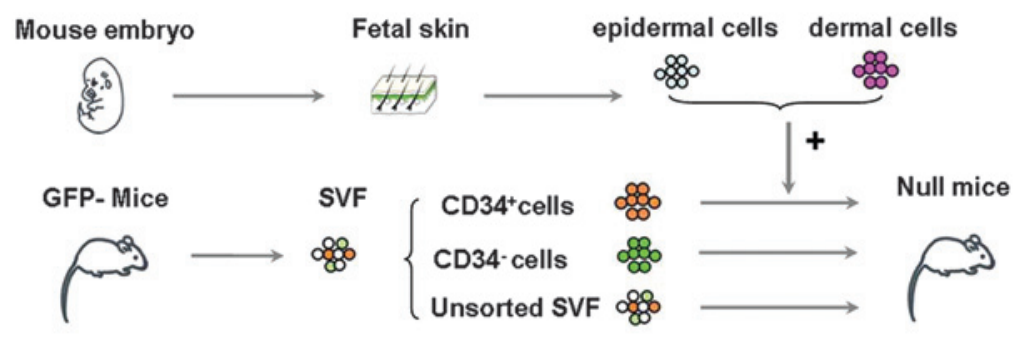

Fresh isolation

Cell Sorting

Cell transplantation

B
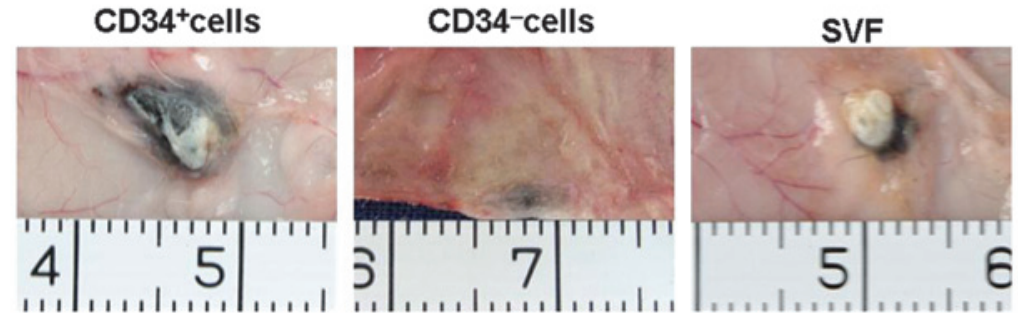

Figure 1. Schematic approach and gross view results of adipose-derived cells in hair morphogenesis. (A) Schematic research plan of skin reconstitution and hair morphogenesis. (B) Gross view of reconstituted skin in three cell groups. GFP-TG mouse, green fluorescence protein transgenic mouse; SVF, stromal vascular fraction.

respectively, and then injected subcutaneously by inserting the pipette tip into the dorsal skin $(n=3)$. After recovery from anesthesia, mice were caged individually and then sacrificed at 3 weeks post-cell transplantation and tissue specimens were harvested and subjected to various studies.

Histological analysis and immunofluorescent staining. For the immunofluorescent analysis, freshly harvested dorsal skin, including implanted tissue, were fixed for $4 \mathrm{~h}$ at room temperature in PBS containing $4 \%$ paraformaldehyde and $10 \%$ glutaraldehyde, and then switched to PBS containing $30 \%$ glutaraldehyde overnight at $4^{\circ} \mathrm{C}$. The fixed tissues were embedded quickly into embedding medium (OCT). The tissues were subsequently frozen and sectioned at the thickness of $10 \mu \mathrm{m}$. Sections were first permeabilized in PBS supplemented with $0.25 \%$ Triton X-100 for 60 min followed by blocking with $10 \%$ goat serum in PBS at room temperature. The sections were then stained with rabbit anti-mouse CD31 (1:50, in PBS containing 1\% BSA, Abcam, Cambridge, UK), rabbit anti-mouse CK15 (1:200, in PBS containing 1\% $\mathrm{BSA}$, Abcam) overnight at $4^{\circ} \mathrm{C}$, followed by the incubation with the secondary antibodies Alexa Fluor 488 or Alexa Fluor 555-conjugated goat anti-rabbit IgG (1:1,000, in PBS containing $1 \%$ BSA, Invitrogen, Eugene, OR, USA) for $30 \mathrm{~min}$ at $37^{\circ} \mathrm{C}$. Fluorescent staining of lipids was performed using LipidTox (1:200 in PBS with 1\% BSA, Invitrogen) as described previously (3). Sections were finally counterstained with DAPI (1:1,000 in PBS with 1\% BSA, Sigma). For histological analysis, the sections were stained with hematoxylin and eosin (H\&E). To analyze the participation rate, section slides were observed under a fluorescent microscope to determine the participation of GFP-labeled cells in the formed tissues of each examined slide.

Quantitative and statistical analyses. The positive and negative slide numbers derived from the groups of hair follicle, blood vessel and fat tissue were counted. Both positive and negative numbers derived from 3 examined specimens of each group were respectively combined, and a Chi-square analysis of the data was performed. A P-value of $<0.05$ was considered to indicate a statistically significant result (Table I).

\section{Results}

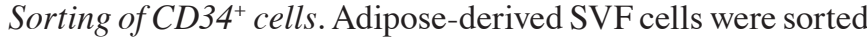
based on antibody cocktail staining. Generally, $6.14 \pm 2.95 \%$ of $\mathrm{CD}_{3}{ }^{+}$cells could be isolated from the gated SVF portion. Further functional characterization revealed that $\mathrm{CD} 4^{+}$cells possessed more potent multi-differentiation potential, stronger proliferative capability and an increased colony forming ability (data not shown) than the cells of the other two groups, indicating that $\mathrm{CD} 4^{+}$cells may represent the mesenchymal stem cells enriched from adipose tissue (ADMSCs).

Participation of $\mathrm{CD} 4^{+}$cells in hair follicle morphogenesis. As previously described (5) and outlined in Fig. 1A, CD $34^{+}$, CD34- and unsorted cells were mixed with mouse fetal keratinocytes and dermal fibroblasts, respectively, and implanted subcutaneously into the back of nude mice. After 3 weeks of in vivo implantation, larger tissue blocks were observed in the $\mathrm{CD}_{34}{ }^{+}$cell group compared with the CD34- and SVF cell groups. Furthermore, more hair follicles were formed in the $\mathrm{CD} 34^{+}$cell group than in the other two groups, whereas an evident fat mass was observed in the SVF cell group (Fig. 1B). These findings suggest that $\mathrm{CD} 34^{+}$cells may further enhance hair follicle morphogenesis compared with CD34- cells and unsorted SVF cells.

As shown in Fig. 2, when analyzed with H\&E staining, $\mathrm{CD} 34^{+}$cells were able to participate in forming hair follicle, blood vessel and fat tissue, as GFP-labeled cells were located at the respective tissues (Fig. 2A), but not in other parts of skin tissue. By contrast, no participation of hair follicle morpho- 
A

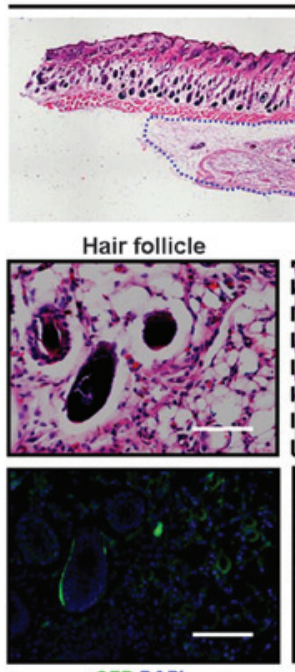

GFP DAPI
CD34+cells

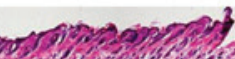

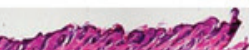

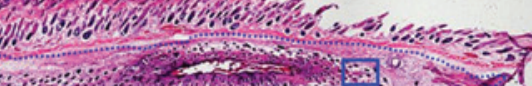

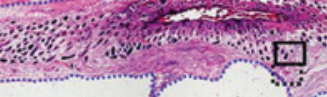

Blood vessel

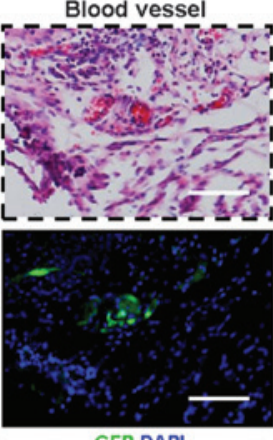

GFP DAPI
B

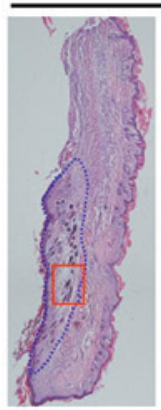

CD34-cells

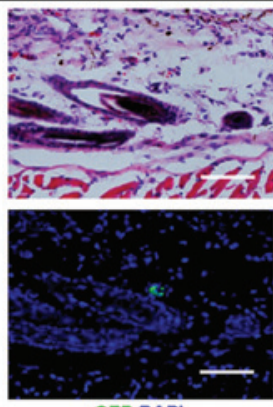

GFP DAPI
C

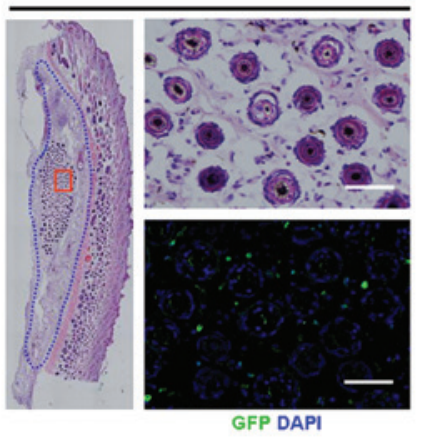

Figure 2. Histological analysis of the participation of adipose-derived cells in different tissue structures in a reconstituted skin model. (A) CD34+ cells participate in forming hair follicles, blood vessels and fat tissue. Top panel, H\&E view of reconstituted skin (highlighted with blue dashed line); middle panel, H\&E images amplified from top panel; bottom panel, immunofluorescent images matched to those of middle panel, green color represents implanted cells. (B) H\&E overview, amplified images and matched immunofluorescent images in the CD34- cell group. (C) H\&E overview, amplified images and matched immunofluorescent images in the unsorted SVF cell group. Scale bars, $100 \mu \mathrm{m}$. SVF, stromal vascular fraction.
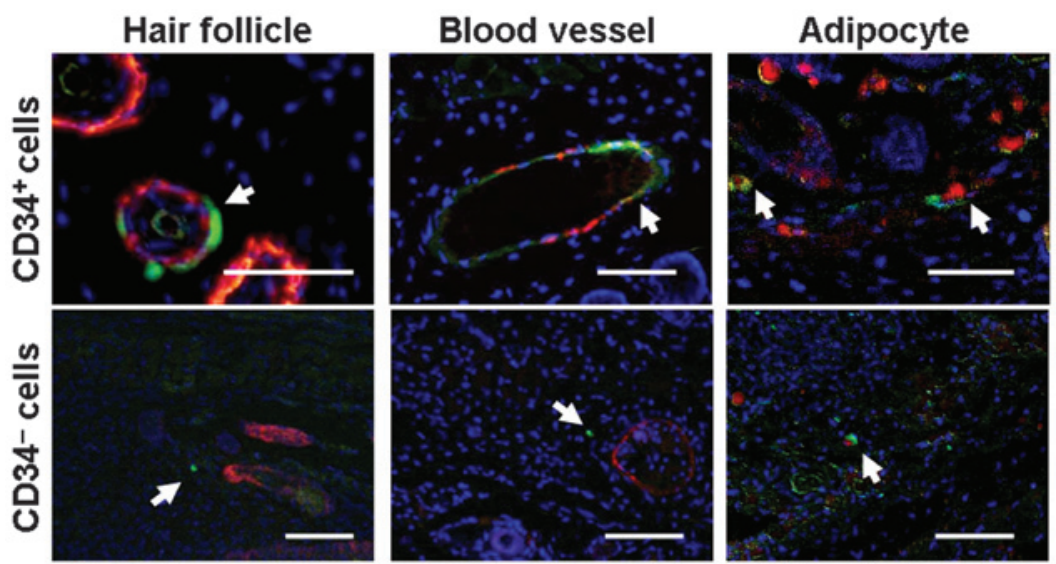

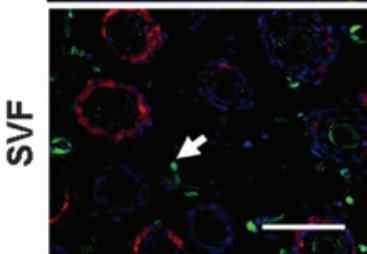

CK15 GFP DAPI

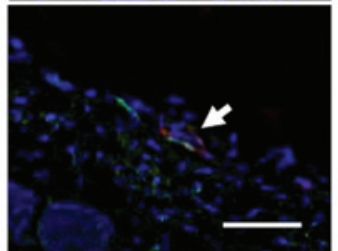

CD31 GFP DAPI

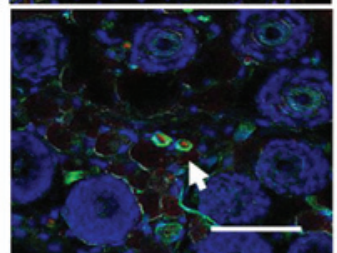

Lipidtox GFP DAPI

Figure 3. Immunofluorescent characterization of differentiated implanted cells in reconstituted skin tissue. CD34 ${ }^{+}$cells (green) were localized at the immediate outlayer of the outer root sheath (CK15 expression in red, top left), indicating participation in dermal sheath formation. Co-localization of green cells and red cells (CD31) was also observed in both CD34+ cells (top middle) and SVF cells (bottom middle) indicating participation in forming blood vessel. Involvement of adipogenesis was observed in all three groups (right panels). Scale bars, $100 \mu \mathrm{m}$. SVF, stromal vascular fraction.

genesis was observed in both CD34- cell and unsorted cell groups (Fig. 2B and C), indicating that there is a difference in differentiation potential among the three groups of cells.

Characterization of differentiated $\mathrm{CD} 4^{+}$cells in developed skin tissue. To further confirm the histological findings, immunofluorescent staining was employed to characterize the formed tissue. Consistent with the histological findings, co-localization of GFP cells and other fluorescent staining indicated that $\mathrm{CD} 34^{+}$cells were capable of participating in the formation of multiple tissues in developed skin (Fig. 3, top panel). Firstly, GFP-positive cells were often observed surrounding the outer root sheath of hair follicles. However, GFP cells (green) were actually located at the immediate outlayer of the CK15-positive cell layer (a marker for the outer root sheath of the hair follicle, red), demonstrating no co-localization to CK15 (Fig. 3, top left). This finding suggests that $\mathrm{CD} 34^{+}$cells may be integrated into the formed dermal 
sheath (DS). In addition, co-localization of GFP cells (green) and CD31 cells (red) demonstrated the participation of CD34 ${ }^{+}$ cells in forming blood vessels (Fig. 3, top middle). Additionally, co-localization of GFP (green) in the cytoplasm with intracellular lipid drops (red) demonstrated the participation of CD34 ${ }^{+}$ cells in forming fat tissue (Fig. 3, top right).

In contrast to $\mathrm{CD} 34^{+}$cells, no involvement of CD34- cells and unsorted SVF cells in hair follicle morphogenesis (Fig. 3, middle left and bottom left) was observed. Although their involvement in fat tissue formation was observable (Fig. 3, middle right and bottom right), involvement in blood vessel formation was only observed in unsorted SVF cells (Fig. 3, middle bottom), but not in CD34- cells (Fig. 3, middle central).

Quantitative analysis of cell participation rate. As shown in Table I, the participation rates for the hair follicle, blood vessel and fat tissue groups were $26.89 \pm 5.86,44.33 \pm 1.82$ and $43.39 \pm 1.90 \%$ for the $\mathrm{CD} 34^{+}$group, $0.00 \pm 0.00,0.00 \pm 0.00$ and $21.47 \pm 7.62 \%$ for the $\mathrm{CD} 34^{+}$group and $0.00 \pm 0.00,32.41 \pm 2.92$, and $86.78 \pm 1.43 \%$ for the SVF group, respectively. Statistical analysis revealed a significant difference in the participation rates among each of the three groups $(\mathrm{P}<0.05)$.

\section{Discussion}

Adult stem cells are derived from various tissues of the body and are able to differentiate and form different types of tissue. Therefore, adult stem cells, such as BMSCs, ASCs, umbilical cord (UC)-MSCs and placenta MSCs are widely explored as potential therapeutic cell sources for tissue and organ regeneration (10-12).

The best-studied adult stem cells are BMSCs and their therapeutic potential has already been shown by reported clinical trials with favorable outcomes $(13,14)$. However, use of BMSCs is limited as harvesting them in large quantities may be harmful to the human body due to loss of hematopoietic stem cells (15). By contrast, ASCs possess several advantages over BMSCs as a promising cell source for tissue regeneration and clinical therapy, including easy access to the source, no harm to the donor and the availability of multiple cell types $(16,17)$.

Nevertheless, the heterogeneity of the ASC population is also a disadvantage for its application in regenerative medicine (7), since the multiple differentiation potential of ASCs is inhibited by the interference of some factors released from different cell types in the pooled population. For example, the difficulty of regenerating homogeneous cartilage-like tissue by pooled adipose-derived cells (18) is likely due to the functional interference to ADMSCs by other cell types. Therefore, purification and relative enrichment with a specific marker would be a reasonable approach for the functional evaluation of ADMSCs.

A study by Rodeheffer et al (8) showed that ADMSCs may be enriched by a series of surface markers including CD29, Sca-1 and CD34. Tang et al (9) showed that ADMSCs may be the vascular pericytes residing in the adipose stromal vascular fraction. Several other studies also demonstrated similar results. Lin et al (19) and Traktuev et al (20) illustrated that ASCs reside at in a perivascular location and CD34 is abundantly expressed in all blood vessels in human adipose tissue. All this evidence suggests that CD34 may be able to serve as a relatively specific marker to enrich ADMSCs. Our study showed that mouse primary $\mathrm{CD} 34^{+} \mathrm{ASC}$ sorted from SVF portion cells are more potent in cell proliferation, clone formation and multi-differentiation compared with $\mathrm{CD}^{-} 4^{-}$and unsorted SVF cells. In addition, the $\mathrm{CD} 34^{+}$cell population also largely overlaps with the $\mathrm{CD} 29^{+}$and $\mathrm{Sca}-\mathrm{I}^{+}$cell populations (data not shown). These results indicate that CD34 may serve as a relatively specific marker for enriching ADMSCs.

Hair follicles consist of concentric epithelial sheaths (hair shaft, inner root sheath, outer root sheath) surrounded by DS that is connected to a dermal papilla (DP) at the base of the follicle (21). In fact, numerous studies have shown that the hair follicle's regenerative cycle, induced by follicular stem cells, is closely associated with adipose tissue and adipogenic lineage cells. For example, the thickness of the intradermal adipocyte layer in the hair follicle active cycle (anagen) increases significantly compared with the thickness of that in the resting phase of the hair cycle $(22,23)$. Furthermore, reduced intradermal adipose tissue in transgenic mice models result in abnormalities in skin structure and function such as hair loss, epidermal hyperplasia and abnormal sebaceous gland function $(4,24,25)$. In particular, Festa et al (3) recently reported that adipose lineage cells, including mature adipocyte and preadipocytes (Lineage-: CD29+:Sca- $1^{+}:$CD $34^{+}:$CD24 ${ }^{+}$), have been defined as skin niche cells that positively regulate hair follicle stem cell activity.

More importantly, hair follicle morphogenesis, involving multiple tissue and cell types, has been employed as a model to test the differentiation potential of adult MSCs. A study by Kataoka et al (5) confirmed that BMSCs were capable of differentiating into various cell types, including epidermal keratinocytes, sebaceous gland cells, follicular epithelial cells, dendritic cells and endothelial cells, using this model. In addition, skin-derived precursors (SKPs) can clonally differentiate into dermal cell types and induce hair follicle morphogenesis (26). Yoo et al (27) showed that UC-MSCs, another type of adult stem cell, is able to differentiate and form DP-like tissues and induce new hair follicles.

In this study, we examined the multi-potency of mouse adipose-derived MSCs purified by CD34 using a reported hair follicle-reconstitution model. The results demonstrated that only $\mathrm{CD}_{3} 4^{+}$cells were able to participate in hair follicle morphogenesis by contributing to the DS formation. Quantitative analysis revealed a participation rate of $27 \%$ in DS structures in the $\mathrm{CD}_{3} 4^{+}$group, whereas a $0 \%$ participation rate was found in the other two groups with a statistically significant difference among the three groups $(\mathrm{P}<0.05)$. A number of studies suggest that DS plays a key role in hair induction. For example, Yamao et al (28) reported that DS formation is critical to the normal development of hairs with hair shafts. In addition, previous studies demonstrating that DS cells and DP cells can be transformed into each other (29), and that lower DS cells can regenerate the DP (30). Moreover, the study by Biernaskie et al (26) also highlighted that SKPs are capable of differentiating into interfollicular dermal cell types in vivo, including DS and DP cells.

This study also revealed that $\mathrm{CD} 34^{+}$cells may be involved in blood vessel formation by differentiating into endothelial cells, besides the differentiation to adipocytes. By contrast, CD34- cells were unable to participate in hair follicle morpho- 
genesis and blood vessel formation. Unsorted SVF may participate in blood vessel formation due to their contained endothelial cells in the pool. However, quantitative analysis revealed there was a significant difference in the participation rate between $\mathrm{CD} 4^{+}$and $\mathrm{SVF}$ groups $(\mathrm{P}<0.01)$. These results, in addition to previous findings $(8,9,19,20)$, indicate that CD34 may serve as a valid marker to enrich ADMSCs.

When compared with BMSCs that participate in the morphogenesis of multiple skin appendages, CD34-enriched adipose cells appear less potent than BMSCs in multiple differentiations, particularly in skin cell differentiation and tissue formation. This finding also agrees with other findings that the differentiation potential varies among different types of adult MSCs, and BMSCs appear to possess better stemness compared with other adult-type MSCs $(15,18)$.

\section{Acknowledgements}

This study was supported by the National Natural Science Foundation of China (30872694, 31170937). The authors also appreciate the technical support from Dr Jingjun Chen, Dr Wanrao Xia, Mr. Demin Ying, Ms. Lijuan Zong and Ms. Juanjuan Wu.

\section{References}

1. Zuk PA, Zhu M, Mizuno H, et al: Multilineage cells from human adipose tissue: implications for cell-based therapies. Tissue Eng 7: 211-228, 2001

2. Gronthos S, Franklin DM, Leddy HA, Robey PG, Storms RW and Gimble JM: Surface protein characterization of human adipose tissue-derived stromal cells. J Cell Physiol 189: 54-63, 2001.

3. Festa E, Fretz J, Berry R, et al: Adipocyte lineage cells contribute to the skin stem cell niche to drive hair cycling. Cell 146: 761-771, 2011.

4. Chen HC, Smith SJ, Tow B, Elias PM and Farese RV Jr: Leptin modulates the effects of acyl CoA:diacylglycerol acyltransferase deficiency on murine fur and sebaceous glands. J Clin Invest 109: 175-181, 2002.

5. Kataoka K, Medina RJ, Kageyama T, et al: Participation of adult mouse bone marrow cells in reconstitution of skin. Am J Pathol 163: 1227-1231, 2003.

6. Li H, Fu X, Ouyang Y, Cai C, Wang J and Sun T: Adult bonemarrow-derived mesenchymal stem cells contribute to wound healing of skin appendages. Cell Tissue Res 326: 725-736, 2006.

7. Yoshimura K, Shigeura T, Matsumoto D, et al: Characterization of freshly isolated and cultured cells derived from the fatty and fluid portions of liposuction aspirates. J Cell Physiol 208: 64-76, 2006.

8. Rodeheffer MS, Birsoy K and Friedman JM: Identification of white adipocyte progenitor cells in vivo. Cell 135: 240-249, 2008

9. Tang W, Zeve D, Suh JM, et al: White fat progenitor cells reside in the adipose vasculature. Science 322: 583-586, 2008.

10. Pittenger MF, Mackay AM, Beck SC, et al: Multilineage potential of adult human mesenchymal stem cells. Science 284: 143-147, 1999.

11. Kuci S, Kuci Z, Latifi-Pupovci H, et al: Adult stem cells as an alternative source of multipotential (pluripotential) cells in regenerative medicine. Curr Stem Cell Res Ther 4: 107-117, 2009.
12. Kakinuma S, Tanaka Y, Chinzei R, et al: Human umbilical cord blood as a source of transplantable hepatic progenitor cells. Stem Cells 21: 217-227, 2003

13. Wakitani S, Imoto K, Yamamoto T, Saito M, Murata N and Yoneda M: Human autologous culture expanded bone marrow mesenchymal cell transplantation for repair of cartilage defects in osteoarthritic knees. Osteoarthritis Cartilage 10: 199-206, 2002.

14. Guo W, Wang H, Zou S, et al: Bone marrow stromal cells produce long-term pain relief in rat models of persistent pain. Stem Cells 29: 1294-1303, 2011

15. Wagner W, Wein F, Seckinger A, et al: Comparative characteristics of mesenchymal stem cells from human bone marrow, adipose tissue, and umbilical cord blood. Exp Hematol 33: 1402-1416, 2005.

16. Aust L, Devlin B, Foster SJ, et al: Yield of human adiposederived adult stem cells from liposuction aspirates. Cytotherapy 6: 7-14, 2004

17. Varma MJ, Breuls RG, Schouten TE, et al: Phenotypical and functional characterization of freshly isolated adipose tissuederived stem cells. Stem Cells Dev 16: 91-104, 2007.

18. Afizah H, Yang Z, Hui JH, Ouyang HW and Lee EH: A comparison between the chondrogenic potential of human bone marrow stem cells (BMSCs) and adipose-derived stem cells (ADSCs) taken from the same donors. Tissue Eng 13: 659-666, 2007.

19. Lin G, Garcia M, Ning H, et al: Defining stem and progenitor cells within adipose tissue. Stem Cells Dev 17: 1053-1063, 2008.

20. Traktuev DO, Merfeld-Clauss S, Li J, et al: A population of multipotent CD34-positive adipose stromal cells share pericyte and mesenchymal surface markers, reside in a periendothelial location, and stabilize endothelial networks. Circ Res 102: 77-85, 2008.

21. Hardy MH: The secret life of the hair follicle. Trends Genet 8: 55-61, 1992.

22. Chase HB, Montagna W and Malone JD: Changes in the skin in relation to the hair growth cycle. Anat Rec 116: 75-81, 1953.

23. Hansen LS, Coggle JE, Wells J and Charles MW: The influence of the hair cycle on the thickness of mouse skin. Anat Rec 210: $569-573,1984$.

24. Jong MC, Gijbels MJ, Dahlmans VE, et al: Hyperlipidemia and cutaneous abnormalities in transgenic mice overexpressing human apolipoprotein C1. J Clin Invest 101: 145-152, 1998.

25. Stone SJ, Myers HM, Watkins SM, et al: Lipopenia and skin barrier abnormalities in DGAT2-deficient mice. J Biol Chem 279: 11767-11776, 2004

26. Biernaskie J, Paris M, Morozova O, et al: SKPs derive from hair follicle precursors and exhibit properties of adult dermal stem cells. Cell Stem Cell 5: 610-623, 2009.

27. Yoo BY, Shin YH, Yoon HH, Seo YK, Song KY and Park JK: Application of mesenchymal stem cells derived from bone marrow and umbilical cord in human hair multiplication. J Dermatol Sci 60: 74-83, 2010.

28. Yamao M, Inamatsu M, Ogawa Y, et al: Contact between dermal papilla cells and dermal sheath cells enhances the ability of DPCs to induce hair growth. J Invest Dermatol 130: 2707-2718, 2010.

29. Tobin DJ, Gunin A, Magerl M, Handijski B and Paus R: Plasticity and cytokinetic dynamics of the hair follicle mesenchyme: implications for hair growth control. J Invest Dermatol 120: 895-904, 2003.

30. McElwee KJ, Kissling S, Wenzel E, Huth A and Hoffmann R: Cultured peribulbar dermal sheath cells can induce hair follicle development and contribute to the dermal sheath and dermal papilla. J Invest Dermatol 121: 1267-1275, 2003. 